Article

\title{
BnaA03.MKK5-BnaA06.MPK3/BnaC03.MPK3 Module Positively Contributes to Sclerotinia sclerotiorum Resistance in Brassica napus
}

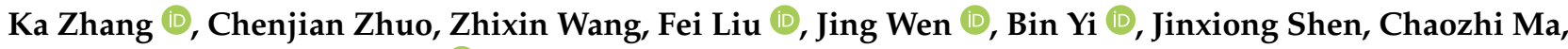 \\ Tingdong Fu and Jinxing Tu*
}

check for

updates

Citation: Zhang, K.; Zhuo, C.; Wang,

Z.; Liu, F.; Wen, J.; Yi, B.; Shen, J.; Ma,

C.; Fu, T.; Tu, J. BnaA03.MKK5-

BnaA06.MPK3/BnaC03.MPK3

Module Positively Contributes to

Sclerotinia sclerotiorum Resistance in

Brassica napus. Plants 2022, 11, 609.

https://doi.org/10.3390/

plants11050609

Academic Editor: Emilie Widemann

Received: 16 January 2022

Accepted: 21 February 2022

Published: 24 February 2022

Publisher's Note: MDPI stays neutral with regard to jurisdictional claims in published maps and institutional affiliations.

Copyright: (C) 2022 by the authors. Licensee MDPI, Basel, Switzerland. This article is an open access article distributed under the terms and conditions of the Creative Commons Attribution (CC BY) license (https:// creativecommons.org/licenses/by/ $4.0 /)$.
National Key Laboratory of Crop Genetic Improvement, College of Plant Science and Technology, National Sub-Center of Rapeseed Improvement in Wuhan, Huazhong Agricultural University, Wuhan 430070, China; zhangka@webmail.hzau.edu.cn (K.Z.); zhuocj@webmail.hzau.edu.cn (C.Z.); wangzhixin1989@webmail.hzau.edu.cn (Z.W.); liufeibio@henu.edu.cn (F.L.); wenjing@mail.hzau.edu.cn (J.W.); yibin@mail.hzau.edu.cn (B.Y.); jxshen@mail.hzau.edu.cn (J.S.); yuanbeauty@mail.hzau.edu.cn (C.M.); futing@mail.hzau.edu.cn (T.F.)

* Correspondence: tujx@mail.hzau.edu.cn; Tel.: +86-027-8728-1819
Abstract: Brassica napus (oilseed rape) is one of the most important oil crops worldwide, but its growth is seriously threatened by Sclerotinia sclerotiorum. The mechanism of oilseed rape response to this pathogen has rarely been studied. Here, it was identified that BnaA03.MKK5 whose expression was induced by $S$. sclerotiorum infection was involved in plant immunity. BnaA03.MKK5 overexpression lines exhibited decreased disease symptoms compared to wild-type plants, accompanied by the increased expression of camalexin-biosynthesis-related genes, including BnPAD3 and BnCYP71A13. In addition, two copies of BnMPK3 (BnA06.MPK3 and BnC03.MPK3) were induced by Sclerotinia incubation, and BnaA03.MKK5 interacted with BnaA06.MPK3/BnaC03.MPK3 in yeast. These interactions were confirmed using in vivo co-immunoprecipitation assays. In vitro phosphorylation assays showed that BnaA06.MPK3 and BnaC03.MPK3 were the direct phosphorylation substrates of BnaA03.MKK5. The transgenic oilseed rape plants including BnaA06.MPK3 and BnaC03.MPK3 overexpression lines and $B n M P K 3$ gene editing lines mediated by CRISPR/Cas9 were generated; the results of the genetic transformation of BnaA06.MPK3/BnaC03.MPK3 indicate that BnMPK3 also has a positive role in Sclerotinia resistance. This study provides information about the potential mechanism of $B$. napus defense against $S$. Sclerotiorum mediated by a detailed BnaA03.MKK5-BnaA06.MPK3/BnaC03.MPK3 module.

Keywords: Brassica napus; Sclerotinia sclerotiorum; mitogen-activated protein kinase; plant defense; molecular mechanism

\section{Introduction}

Brassica napus (genome AACC) is an allotetraploid species derived from the natural hybridization of diploid ancestors Brassica rapa (genome AA) and Brassica oleracea (genome CC) about 7500 years ago; it contains 19 pairs of chromosomes (A01 A10 and C01 C09) [1]. In nature, oilseed rape is frequently attacked by Sclerotinia sclerotiorum, which causes large losses of crop yield [2,3]. S. sclerotiorum is a typical necrotrophic fungal pathogen with a wide range of hosts, including many important economic crops such as oilseed rape, peanut, soybean, and sunflower [4].

When challenged by pathogens, the innate immune system of plants is activated. The plant innate immune system has evolved into two branches, pathogen-associated molecular pattern (PAMP)-triggered immunity (PTI) and effector-triggered immunity (ETI) [5,6]. Both PTI and ETI can trigger the activation of kinase cascades mediated by the mitogen-activated protein kinase (MAPK) class [7,8]. The basic MAPK cascade consists of three graded kinases (MAPKKKs (MEKKs), MAPKKs (MKKs) and MAPKs (MPKs)). MPKs are phosphorylated 
and activated by upstream MPK kinases (MKKs), and MKKs are activated by upstream MKK kinases (MEKKs) through the phosphorylation of conserved Ser/Thr in the activation loop ((S/T)XXXXX(S/T)) of plant MKKs [9]. In addition, MKKs phosphorylate MPKs by acting on Thr/Tyr residues in the activation motif (Thr-X-Tyr) of MPKs, while the common docking (CD) site at the carboxyl-terminal of MPKs includes two adjacent acidic amino acid residues (Asp and Glu), which are essential for the interaction with MKKs [10].

In the Arabidopsis thaliana genome, there are $60 \mathrm{MEKKs}, 10 \mathrm{MKKs}$, and $20 \mathrm{MPKs}$ [11]. MEKK1 and MEKK2 are reported to be involved in the salicylic-acid-mediated plant defense response [12]. MKKs can be further divided into four groups (A D), in which MKK1/2/ 6 belongs to group A, group B only contains MKK3, MKK4/5 belongs to group C, and group D contains MKK7/8/9/10 [10]. According to the variation of $X$ in the conserved Thr-X-Tyr tripeptide motif, MPKs can be divided into two categories (TEY and TDY), in which TEY can be divided into three groups $(\mathrm{A} \sim \mathrm{C})$, and TDY is classified into group $\mathrm{D}$ [9]. The well-studied members MPK3/6 belongs to group A, and MPK4 belongs to group B. MKK1/2 acts upstream of MPK4, and the MKK1/2-MPK4 module works in plant defense against pathogens and tolerance to low temperature and salt stress [12,13]. MKK3 participates in jasmonic acid signal transduction by acting on MPK6 [14]. The two kinases MKK4/5 in group C are involved in plant innate immunity $[15,16]$ and are also related to the development of stomatal and floral organs by phosphorylating MPK3/6 [17,18]. The MKK9-MPK6 module promotes leaf senescence [19].

Many attempts have been made to understand the mechanism of oilseed rape response to Sclerotinia infection. Oxalic acid secreted by S. sclerotiorum is considered to be one of the main causes of host disease [20]; Liu et al. found that the heterologous overexpression of barley oxalate oxidase coding the BOXO gene in oilseed rape can partially enhance Sclerotinia resistance by reducing the oxalate level and increasing the hydrogen peroxide level in transgenic lines [21]. Polygalacturonase secreted by S. sclerotiorum is another pathogenic factor [22]; overexpression of the gene encoding rice polygalacturonase inhibitor protein 2 (OsPGIP2) in oilseed rape can improve the stem resistance of $B$. napus to S. sclerotiorum [23]. BnWRKY33 is a positive regulatory factor in Sclerotinia response. BnWRKY33-overexpression lines show less damage than wild-type (WT) plants [24,25]. Wang et al. examined the role of BnMPK3, BnMPK4, and BnMPK6 in Sclerotinia resistance and found that the overexpression of these genes could enhance Sclerotinia resistance in transgenic lines [26-28]. Camalexin, the major phytoalexin in Arabidopsis and Brassicaceae species, is closely related to defense against Sclerotinia sclerotiorum and Botrytis cinerea [29,30]. When plants are infected by these pathogens, camalexin-biosynthesis-related genes are induced, and phytoalexins are accumulated. The high expression of camalexin biosynthetic genes contributes to strong resistance to fungal pathogens. Two cytochrome P450 enzymes coding genes, CYP71A13 and CYP71B15 (PAD3), are involved in camalexin biosynthesis [31].

$S$. sclerotiorum causes great damage to the growth of $B$. napus, but our knowledge about the immune mechanism of oilseed rape against this pathogen is limited; in particular, the role of the MAPK cascade involved in is still obscure. In this study, we provide direct evidence that BnaA03.MKK5 and BnaA06.MPK3/BnaC03.MPK3 act as a module and positively contribute to Sclerotinia resistance.

\section{Results}

\subsection{BnaA03.MKK5 Participated in Response to S. sclerotiorum Infection}

B. napus and model plant $A$. thaliana are Cruciferae members [32]; S. sclerotiorum and $B$. cinerea, two closely related necrotrophic fungal pathogens, have large-scale genomic collinearity, share more than $80 \%$ amino acid identity, and are similar in terms of their pathogenic mechanisms and metabolites [22]. In order to explore the response mechanism of oilseed rape to $S$. sclerotiorum infection, we focused on the MAPK cascade, including AtMKK5, the role of which in response to biotic and abiotic stress, especially in defense against B. cinerea in A. thaliana, has been well documented [15]. AtMKK4 and AtMKK5 are similar in sequence and functionally redundant [11]. We identified four MKK4 copies and 
four MKK5 copies in the B. napus genome via homologous alignment with AtMKK4 and AtMKK5, respectively, and found that these BnMKK4 and BnMKK5 copies were highly similar in sequence (Figure S1), suggesting that they may have redundant potential functions. Nevertheless, among these BnMKK4/BnMKK5 copies, a paralog named BnMKK4 (GenBank accession No. JF268686) was reported to be involved in low-temperature and salt stress response in B. napus [33]. In fact, JF268686 is more similar to AtMKK5 (it shows an identity of $85 \%$ with AtMKK5 and $71 \%$ with AtMKK4 at the amino acid level), and we cloned this gene in oilseed rape (named BnaA03.MKK5) according to the JF268686 sequence (Figure 1A). BnaA03.MKK5 contains a large amount of conserved motifs compared with JF268686 (99.7\% identity), AtMKK5 (86\% identity) and AtMKK4 (72\% identity) (Figure 1A), and BnaA03.MKK5 could be rapidly induced by S. Sclerotiorum (Figure 1B). The transcript abundance of BnaA03.MKK5 accumulated $12 \mathrm{~h}$ after inoculation with Sclerotinia, remained at a high level $24 \mathrm{~h}$ after infection, and then decreased. This result suggests that BnaA03.MKK5 may be involved in the early response to S. sclerotiorum in oilseed rape. To confirm the role of BnaA03.MKK5 in defense, we generated an active mutant of BnaA03.MKK5 (named BnaA03.MKK5 ${ }^{\mathrm{DD}}$ ), by mutating the conserved Ser/Thr in the activation loop to Asp (Figure 1C). Then, we expressed BnaA03.MKK5 and BnaA03.MKK5 ${ }^{\mathrm{DD}}$ driven by CaMV35S (cauliflower mosaic virus 35S promoter) in Nicotiana benthamiana leaves. It was found that constitutively activated BnaA03.MKK5 ${ }^{\mathrm{DD}}$, but not BnaA03.MKK5, induced hypersensitive response cell death (Figure 1D). Hypersensitive response cell death is frequently associated with plant disease resistance $[16,34]$. These data indicate that BnaA03.MKK5 responds to $S$. sclerotiorum infection and may have a role when plants are challenged by this pathogen.
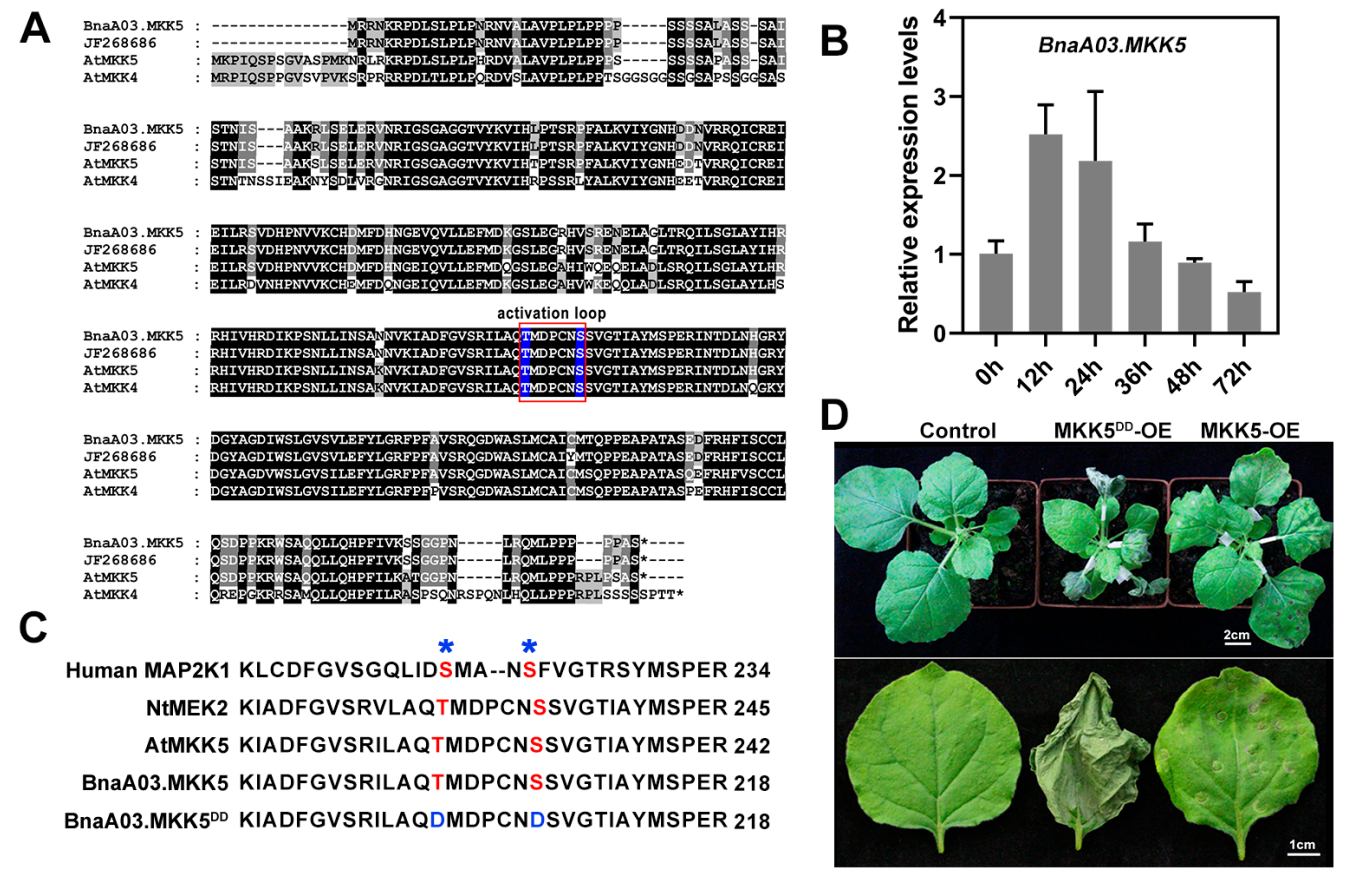

Figure 1. BnaA03.MKK5 is involved in defense response. (A) Sequence alignment of BnaA03.MKK5, JF268686, AtMKK5, and AtMKK4. The conserved amino acid residues are shown in black shading and similar residues are displayed in gray shading. The key motif activation loop is marked with red square frame, and conserved Ser/Thr is highlighted in blue. At, Arabidopsis thaliana; Bna, Brassica napus. (B) Induced expression profiles of BnaA03.MKK5 were identified at $0 \mathrm{~h}, 12 \mathrm{~h}, 24 \mathrm{~h}, 36 \mathrm{~h}, 48 \mathrm{~h}$, and $72 \mathrm{~h}$ after inoculation with S. sclerotiorum. Data are shown as means $\pm \mathrm{SD}(n=3)$. (C) Diagram of the conserved Ser/Thr residues in the activation loop of BnaA03.MKK5. The target sites were detected via alignment to the homologous sequences in other species. BnaA03.MKK5 ${ }^{\mathrm{DD}}$, conserved Ser/Thr mutated to Asp. Nt, Nicotiana benthamiana. (D) Constitutively active BnaA03.MKK5 ${ }^{\mathrm{DD}}$ induced hypersensitive response cell death. Hypersensitive response cell death induced by overexpression of 
constitutively active BnaA03.MKK5 ${ }^{\mathrm{DD}}\left(\mathrm{MKK} 5^{\mathrm{DD}}-\mathrm{OE}\right)$ in $N$. benthamiana leaves. MKK5-OE, plants infiltrated with Agrobacterium carrying BnaA03.MKK5 overexpression driven by CaMV35S; MKK5 ${ }^{\mathrm{DD}}$. OE, plants infiltrated with Agrobacterium carrying BnaA03.MKK5 ${ }^{D D}$ overexpression driven by CaMV35S; control, without injection. Scale bars, $2 \mathrm{~cm}$ (top image), $1 \mathrm{~cm}$ (bottom image).

\subsection{BnaA03.MKK5 Overexpression Lines Showed Enhanced Resistance to S. sclerotiorum in B. napus}

To further explore the biological function of BnaA03.MKK5 in defense against Sclerotinia in oilseed rape, we generated BnaA03.MKK5 overexpression (MKK5-OE) transgenic lines. The full-length coding sequence (CDS) of BnaA03.MKK5 driven by CaMV35S was introduced into oilseed rape plants, and seven independent transgenic lines with different expression levels were selected for analysis based on qRT-PCR analysis (Figure 2A). Leaves from approximately 6-week-old $\mathrm{T}_{2}$ generation MKK5-OE lines and WT plants were used for inoculation with S. sclerotiorum. We measured the lesion area of these infected leaves after $48 \mathrm{~h}$ of inoculation (Table S1). The overexpression lines with high expression of BnaA03.MKK5 tended to show milder lesions than the untransformed WT plants after $48 \mathrm{~h}$ of inoculation with $S$. Sclerotiorum (Figure 2B,C), indicating that MKK5-OE lines delay lesion expansion compared with WT plants. We also analyzed the pathogen-induced expression of camalexin biosynthetic genes, including BnPAD3 and BnCYP71A13 in two typical MKK5-OE lines after inoculation with S. Sclerotiorum for $24 \mathrm{~h}$ and $36 \mathrm{~h}$. In contrast to WT plants, the MKK5-OE lines showed increased transcript accumulation of BnPAD3 and $B n C Y P 71 A 13$ (Figure 2D,E). These results show that in oilseed rape, the overexpression of BnaA03.MKK5 significantly enhances plants' ability to resist the invasion of pathogens when being attacked by $S$. sclerotiorum.

A

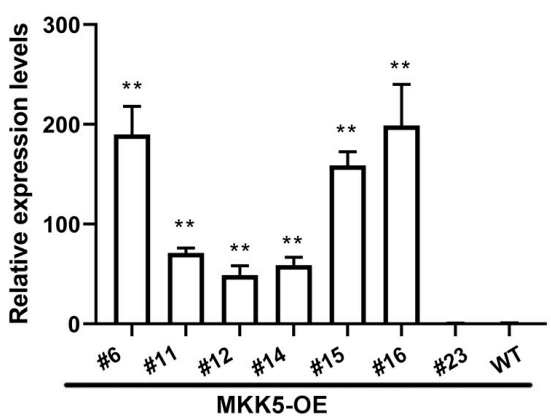

B

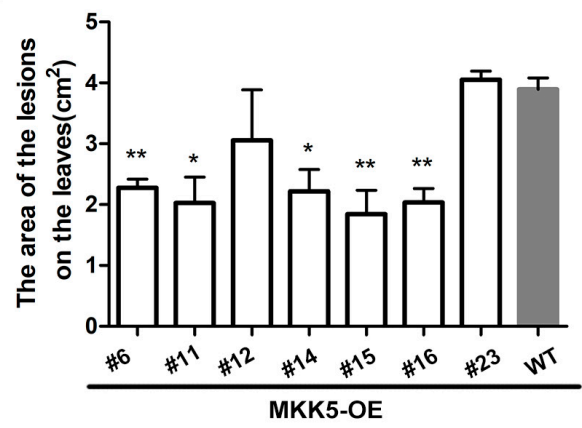

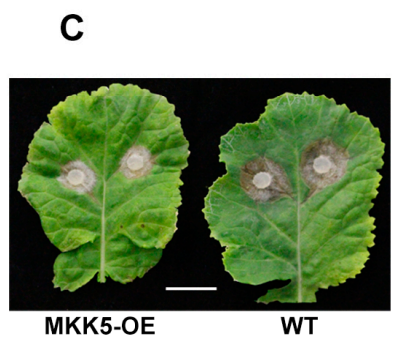

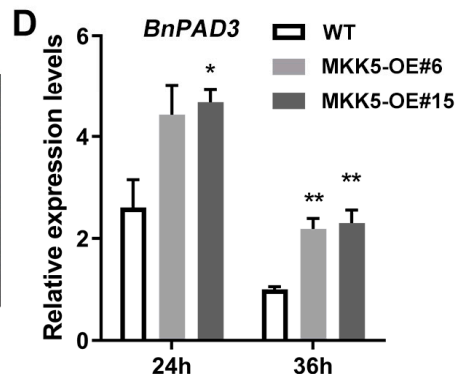

E

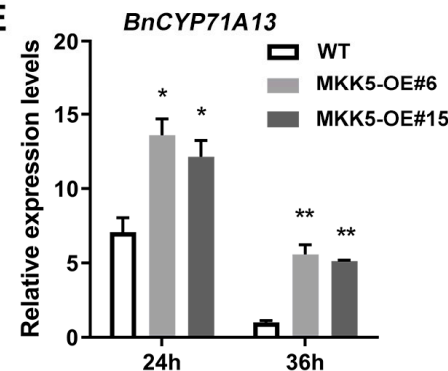

Figure 2. Overexpression of BnaA03.MKK5 enhances $S$. sclerotiorum resistance in B. napus. (A) qRTPCR analysis of BnaA03.MKK5 overexpression (MKK5-OE) lines. The expression in wild-type (WT) plants is used as the control. (B,C) Statistical analysis of lesion areas of MKK5-OE lines after $48 \mathrm{~h}$ of inoculation, and lesion imaging of representative lines. Scale bar, $2 \mathrm{~cm}$. (D,E) Relative expression levels of BnPAD3 and BnCYP71A13 in MKK5-OE lines and WT plants after inoculation for $24 \mathrm{~h}$ and $36 \mathrm{~h}$. In all charts, data are shown as means $\pm \mathrm{SD}(n=3)$. Asterisks indicate significant differences compared with WT plants at corresponding time points $\left(t\right.$-test, $\left.{ }^{*} p<0.05,{ }^{* *} p<0.01\right)$. 


\subsection{Sequence Analysis of MPK3 in B. napus}

Plant MAPK-cascade-mediated signaling is an essential event that occurs in response to pathogens. MKKs function by phosphorylating and activating their downstream MPKs $[9,11]$. Among the studies concerning the MAPK cascade, most of the elucidated MAPK signaling pathways are related to MAPK3/6 [11]. MAPK3/ 6 can be activated by multiple upstream kinases such as MKK3/4/5/9, and then are involved in developmental processes, biotic stress responses, and abiotic stress responses. In A. thaliana, the MAPK cascade signaling pathway mediated by MKK4/MKK5-MAPK3/MAPK6 module has been reported to play an important role in plant immunity $[15,35,36]$.

To explore the mechanism of BnaA03.MKK5 in Sclerotinia resistance, we considered BnaMPK 3 as its potential substrate. Through sequence alignment and the homology cloning approach, we identified two orthologs of AtMPK3 in oilseed rape, which were located on chromosomes A06 and C03, respectively, namely BnaA06.MPK3 and BnaC03.MPK3. There was only one ortholog of AtMPK3 in B. rapa, named BraA06.MPK3; only one ortholog of AtMPK3 was identified in B. oleracea, named BolC03.MPK3. Sequence analysis of MPK3 in B. napus, B. rapa, B. oleracea, and $A$. thaliana showed that the amino acid sequences of these MPK3 members were almost identical (they shared over $94 \%$ identity). These Brassica MPK3, like AtMPK3, contained conserved TEY domains and a common docking site (Figure 3A). Phylogenetic analyses were conducted to investigate the evolutionary relationship of BnaA06.MPK3/BnaC03.MPK3 to MPK3 members of other representative species (Figure 3B). BnaA06.MPK3 was more closely related to BraA06.MPK3, and BnaC03.MPK3 was more closely related to BolC03.MPK3. That is, BnaA06.MPK3 inherited from the copy in B. rapa, and BnaC03.MPK3 inherited from the copy in B. oleracea. BnaA06.MPK3/BnaC03. MPK3 showed a closer genetic relationship with AtMPK3 and AtMPK6, indicating that BnaA06.MPK3/BnaC03.MPK3 may have similar biological functions with these two kinases (Figure 3B).

A

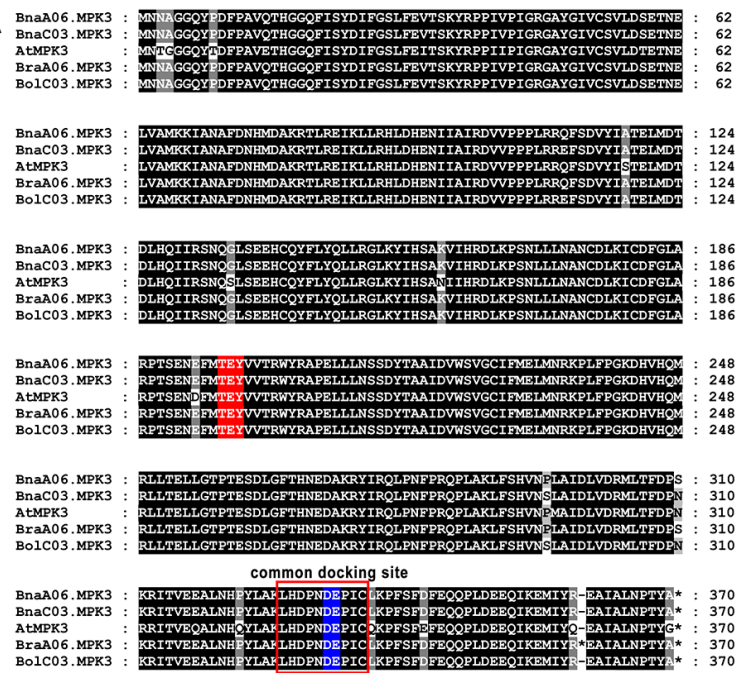

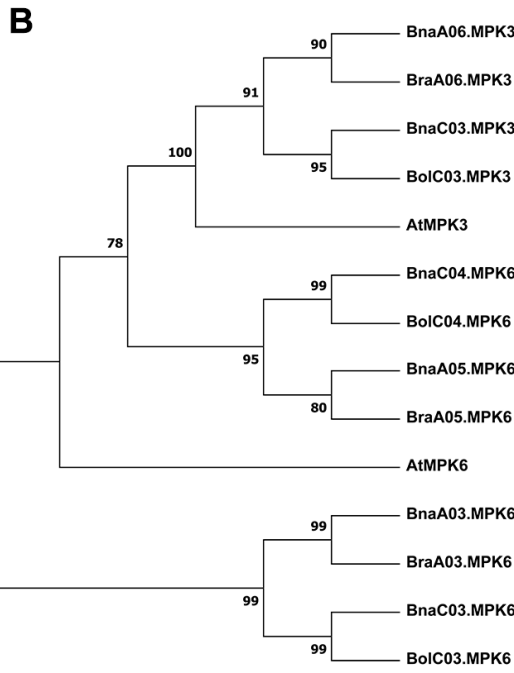

Figure 3. Sequence alignment and phylogenetic tree construction of Brassica MPK3. (A) Amino acid sequence alignments of identified MPK3 copies in Brassica and Arabidopsis. The conserved amino acid residues are shown in black shading and similar residues are displayed in gray shading. TEY motif is marked with red; common docking site is marked with red square frame, and two adjacent acidic residues are highlighted in blue. Bra, Brassica rapa; Bol, Brassica oleracea. (B) Phylogenetic analysis showing the relationship between each copy of B. napus MPK3 and MPK3/MPK6 in Brassica species, as well as MPK3/MPK6 in Arabidopsis. 


\subsection{BnaA06.MPK3 and BnaC03.MPK3 Are the Phosphorylation Substrate of BnaA03.MKK5}

qRT-PCR was performed to determine the expression profiles of BnaA06.MPK3 and BnaC03.MPK3 in WT oilseed rape leaves infected with S. sclerotiorum within $72 \mathrm{~h}$. BnaA06.MPK3 and BnaC03.MPK3 could be significantly induced by S. Sclerotiorum; the expression levels peaked at $24 \mathrm{~h}$ after inoculation and then decreased, but the levels still remained high (Figure 4A,B). These data suggest that BnaA06.MPK3 and BnaC03.MPK3, similar to BnaA03.MKK5, are involved in the defense mechanism against $S$. sclerotiorum.

A

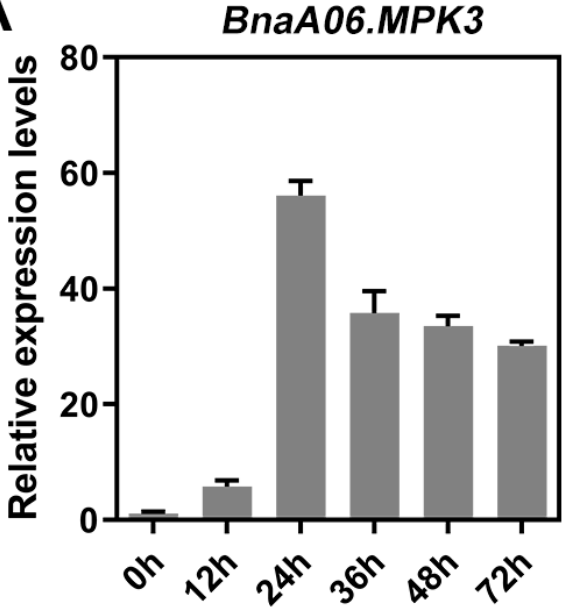

B

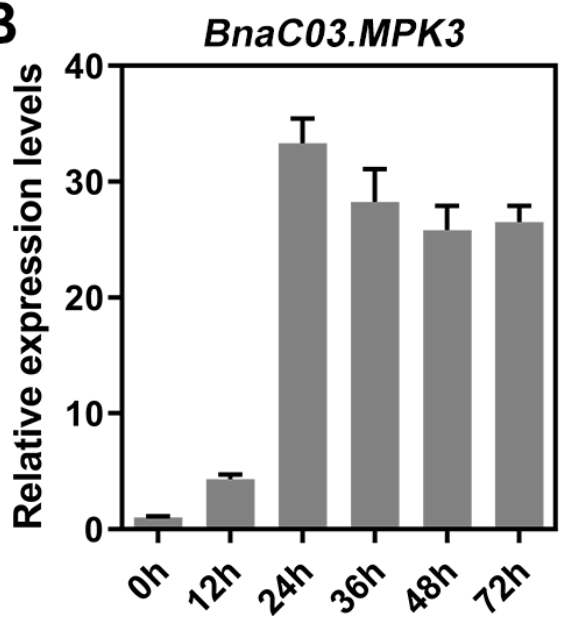

Figure 4. Expression analysis of two BnMPK3 copies in response to Sclerotinia infection. Induced expression profiles of BnaA06.MPK3 (A) and BnaC03.MPK3 (B) were identified at $0 \mathrm{~h}, 12 \mathrm{~h}, 24 \mathrm{~h}, 36 \mathrm{~h}$, $48 \mathrm{~h}$, and $72 \mathrm{~h}$ after inoculation with $\mathrm{S}$. sclerotiorum. Data are shown as means $\pm \mathrm{SD}(n=3)$.

To determine whether BnaA06.MPK3/BnaC03.MPK3 and BnaA03.MKK5 work as a module to participate in Sclerotinia resistance, we first conducted assays to examine the interaction between these kinases. In $\mathrm{Y} 2 \mathrm{H}$ assays, the full-length CDS of BnaA03.MKK5 was inserted into the pGADT7 vector, and BnaA06.MPK3/BnaC03.MPK3 was cloned into the pGBKT7 vector. Yeast cells transformed with the combination of a pGBKT7 empty vector, and AD-MKK5 (BD/AD-MKK5) grew normally on selective dropout medium SD/-Trp Leu, but not on SD/-Trp Leu His Ade. However, when replacing the pGBKT7 empty vector with BD-MAPK3-A6 or BD-MAPK3-C3, the yeast cells could grow normally, even on selective dropout medium SD/-Trp Leu His Ade. In addition, the combination of BD-MAPK3A6 / AD and BD-MAPK3-C3/AD could not grow on SD/-Trp Leu His Ade, indicating that BnaA06.MAPK3/BnaC03.MAPK3 has no self-activation in the Y2H system (Figure 5A). These results suggest that BnA03.MKK5 interacts with BnaA06.MAPK3/BnaC03.MAPK3. The interactions were further confirmed using co-immunoprecipitation (CoIP) assays, in which BnaA03.MKK5-FLAG, BnaA06.MPK3-GFP and BnaC03.MPK3-GFP fusion proteins were coexpressed in N. benthamiana leaves (Figure 5B). BnaA03.MKK5-FLAG coimmunoprecipitated with BnaA06.MPK3-GFP or BnaC03.MPK3-GFP instead of the negative control, indicating that BnaA03.MKK5 can form a protein complex with BnaA06.MAPK3/ BnaC03.MAPK3 in vivo. Next, in vitro phosphorylation assays were performed to determine whether BnaA06.MAPK3/BnaC03.MAPK3 could be phosphorylated directly by upstream BnaA03.MKK5. We expressed GST-tagged BnaA06.MPK3/BnaC03.MPK3 fusion protein in E. coli and purified the protein samples with GST beads. The phosphorylation signal was only detected when BnaA06.MPK3/BnaC03.MPK3 was incubated with BnaA03.MKK5 ${ }^{\mathrm{DD}}$ (Figure 5C). Taken together, the in vitro and in vivo results indicate that BnaA03.MKK5 physically interacts with and phosphorylates BnaA06.MPK3 or BnaC03.MPK3. 
A

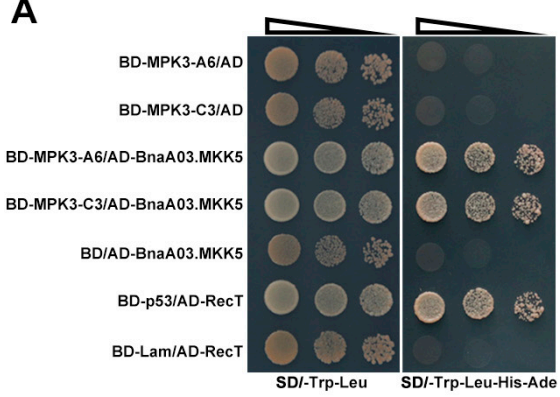

B

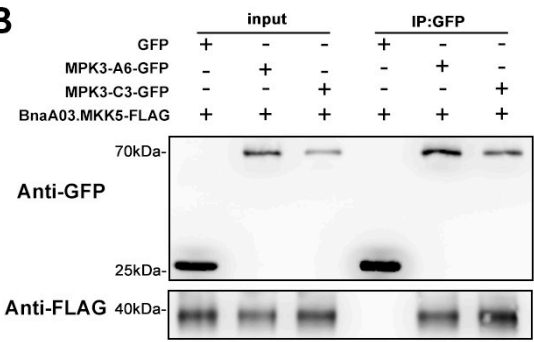

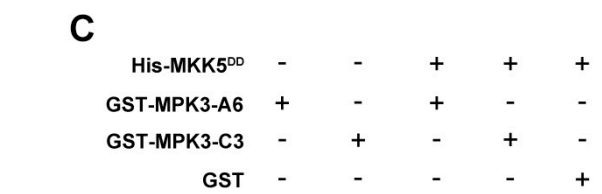
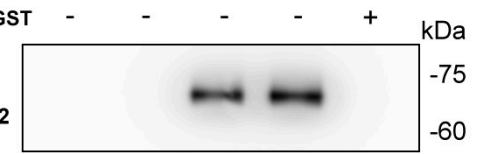

Anti-Phospho-p44/42
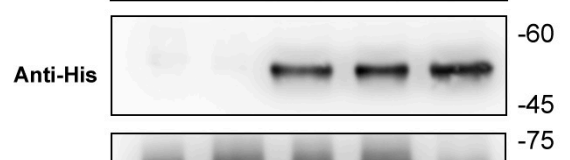
75

Anti-GST

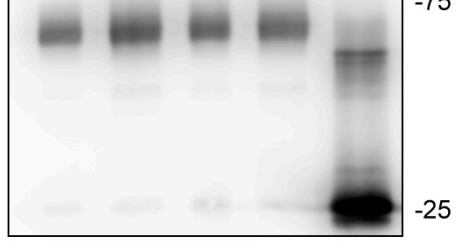

Figure 5. Identification of the interaction between BnaA03.MKK5 and BnaA06.MPK3/BnaC03.MPK3. (A) Yeast two-hybrid (Y2H) to detect the interaction between BnaA03.MKK5 and BnaA06.MPK3/ BnaC03.MPK3. BD-p53/AD-RecT, positive control; BD-Lam/AD-RecT, negative control. (B) Coimmunoprecipitation (CoIP) assays to confirm the interaction between BnaA03.MKK5 and BnaA06.MPK3/BnaC03.MPK3 in planta. (C) In vitro phosphorylation detection of BnaA03.MKK5 on BnaA06.MPK3/BnaC03.MPK3.

\subsection{BnaA06.MPK3/BnaC03.MPK3 Positively Contribute to S. sclerotiorum Resistance in B. napus}

We generated transgenic oilseed rape plants with BnaA06.MPK3- and BnaC03.MPK3overexpression driven by CaMV35S, BnaMPK3 knockout mediated by CRISPR/Cas9. Three independent BnaA06.MPK3 overexpression (MPK3-A6-OE) lines and three independent BnaC03.MPK3 overexpression (MPK3-C3-OE) lines with more than 10-fold increases in expression were used for further analysis (Figure 6A,B). In addition, three independent homozygous mutants with different editing types in the genome region of BnaA06.MPK3 and BnaC03.MPK3 were established for comparison with WT plants (Figure 6C). The conserved amino acid motif TDY/TEY of MAPKs is phosphorylated by MKKs, and the common docking (CD) site located in the carboxyl-terminal of MAPKs includes two adjacent acidic residues (D and E) that are crucial for the interaction with MKKs [10]. The insertions/deletions mediated by CRISPR/Cas9 lead to out-of-frame mutation and the destruction of key domains of target genes. CR\#44 carried a 1-bp insertion in sgRNA1 of A06, a 1-bp insertion in sgRNA2 of A06, and a 4-bp deletion in sgRNA1 of C03; CR\#49 carried a 4-bp deletion in sgRNA1 of A06, a 1-bp insertion in sgRNA2 of A06, a 1-bp deletion in sgRNA1 of C03, and a 1-bp insertion in sgRNA2 of C03. CR\#61 carried a 4-bp deletion in sgRNA1 of A06, a 9-bp deletion in sgRNA2 of A06, a 28-bp deletion in sgRNA1 of C03, and a 1-bp deletion in sgRNA2 of C03. After $48 \mathrm{~h}$ of inoculation with S. sclerotiorum, it was determined that the lesion areas of both BnaA06.MPK3 and BnaC03.MPK3 overexpression lines were smaller than those in WT plants. However, the knockout lines showed a decrease in pathogen resistance (Figure 6D,E and Table S1). The expression of BnPAD3 and BnCYP71A13 in BnaA06.MPK3 and BnaC03.MPK3 overexpression lines were much higher than those in WT plants and remained at a high level with the invasion of pathogens. In BnMPK3 knockout lines, the expressions of these genes did not change much and were at a very low level (Figure $6 \mathrm{~F}, \mathrm{G}$ ). These results show that in B. napus, BnaA06.MPK3/BnaC03.MPK3 has a positive role in inhibiting the damage of pathogens to plants. 

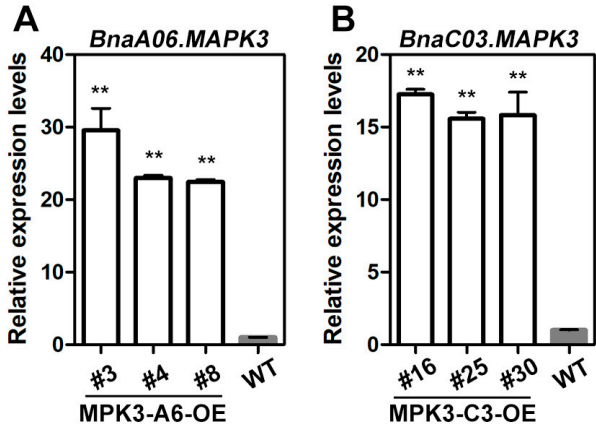

D
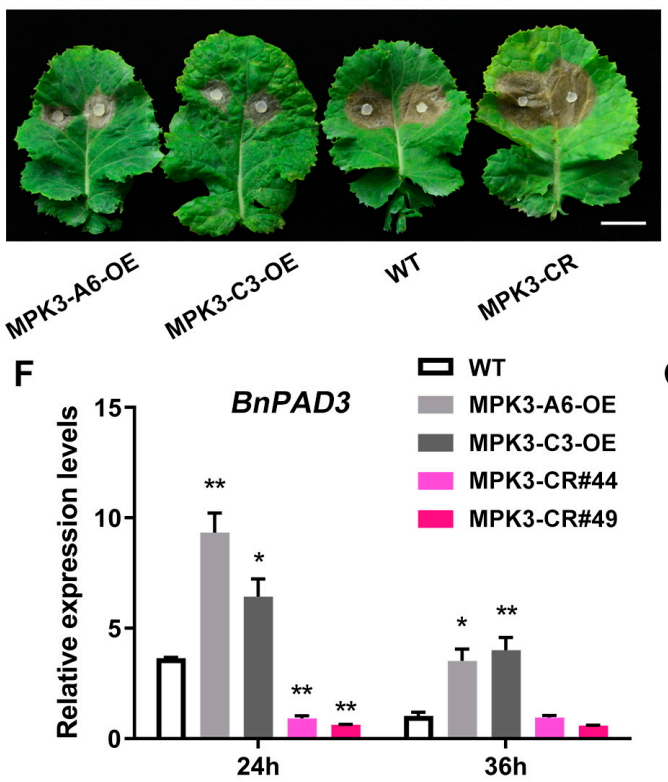

C

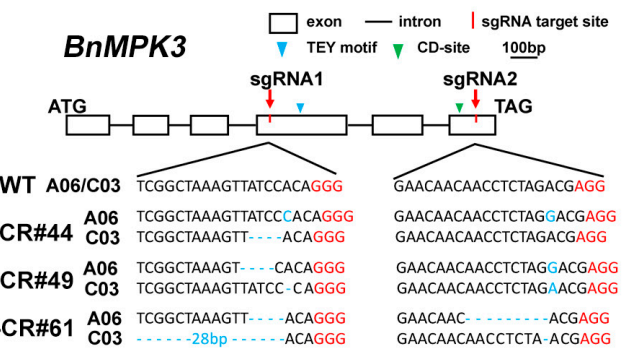

E

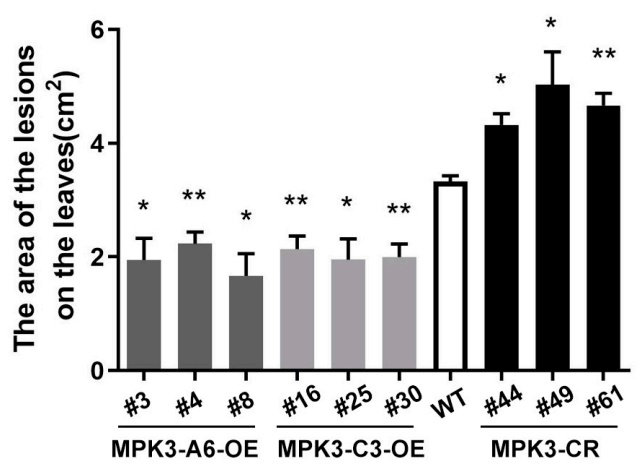

G

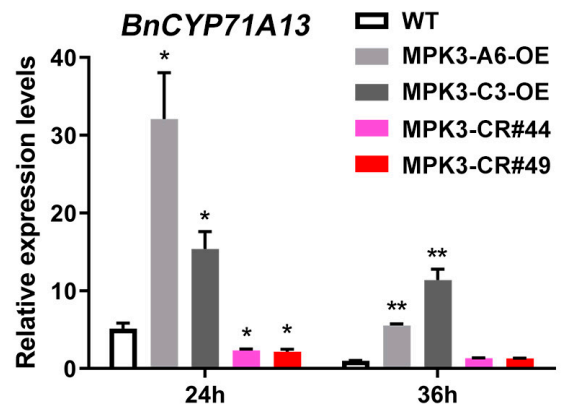

Figure 6. Contribution of BnMPK3 to S. sclerotiorum resistance in B. napus. (A,B) BnaA06.MPK3 overexpression (MPK3-A6-OE) lines (A) and BnaC03.MPK3 overexpression (MPK3-C3-OE) lines (B) established using qRT-PCR analysis. The expression in WT plants as control. Data are shown as means $\pm \mathrm{SD}(n=3)$. Asterisks indicate significant differences compared with WT plants $(t$-test, ** $p<0.01$ ). (C) A schematic diagram to show the characterization of the BnMPK3 exon/intron sequence and the target sites of sgRNAs. The edit types of three independent homozygous mutants (MPK3-CR\#44, 49, and 61) are shown. The protospacer-adjacent motifs (PAM) are marked in red. TEY motif, phosphorylated by MKKs; CD-site, interacts with MKKs; ATG, start codon; TAG, stop codon. (D) Photographs of the lesions of detached leaves from BnMPK3 transgenic lines and WT plants in approximately 4 weeks when inoculated with $7 \mathrm{~mm} \mathrm{~S}$. sclerotiorum hyphae agar block at $22{ }^{\circ} \mathrm{C}$ for $48 \mathrm{~h}$. Scale bar, $2 \mathrm{~cm}$. (E) Statistical analysis of the lesion areas of BnMPK3 transgenic lines and WT plants after $48 \mathrm{~h}$ inoculation. Data are shown as means $\pm \mathrm{SD}(n=3)$. Asterisks indicate significant differences compared with WT ( $t$-test, $\left.{ }^{*} p<0.05,{ }^{* *} p<0.01\right)$. (F,G) Relative expression levels of two camalexin biosynthesis-related genes, BnPAD3 and BnCYP71A13, in BnMPK3 transgenic lines and WT plants after $S$. sclerotiorum inoculation for $24 \mathrm{~h}$ or $36 \mathrm{~h}$. Data are shown as means \pm SD $(n=3)$. Asterisks indicate significant differences compared with $\mathrm{WT}$ at corresponding time points $\left(t\right.$-test, $\left.{ }^{*} p<0.05, * * p<0.01\right)$.

\section{Discussion}

S. sclerotiorum is one of the most harmful diseases to oilseed rape growth. Great efforts have been made to identify the quantitative genetic loci (QTL) defense against Sclerotinia in oilseed rape [37-39]. No germplasm in oilseed rape, or even in all Brassicaceae species, is immune to Sclerotinia, which has made the propagation of resistant varieties less effective for decades, and disease control in oilseed rape primarily relies 
on partially resistant lines [23,39]. Moreover, little progress has been made with regard to the identification of resistant germplasms and genes in B. napus, making it difficult to study the interaction between oilseed rape and Sclerotinia, and the potential molecular mechanisms of oilseed rape response to this pathogen are still poorly understood. As there is no resistance $(R)$ gene in oilseed rape, the plant's innate immune system may be the primary solution to defense against $S$. sclerotiorum for oilseed rape. The MAPK cascade has a significant role in response to pathogens. In this study, we found that the BnaA03.MKK5BnaA06.MPK3/BnaC03.MPK3 module positively contributes to S. sclerotiorum resistance in B. napus. Unfortunately, the MKK5-MPK3 cascade is conserved in the stress response, which means this study lacks novelty. However, it is the first work to identify a detailed MAPK cascade module in Sclerotinia resistance in oilseed rape, which provides new evidence that could reveal the complex response mechanism of oilseed rape defense against Sclerotinia. The results of this study also suggest that the transgenic manipulation of factors associated with the innate immune system provides alternative approaches for the creation of diverse oilseed rape varieties with Sclerotinia resistance.

Four copies of MKK5 were identified in the B. napus genome, located on chromosomes A01, A03, C01, and C03, respectively, and four copies of MKK4 were found on chromosomes A05, A08, C03, and C06 in the B. napus genome. In oilseed rape, these MKK4 and MKK 5 copies exhibited sequence identity and contained a typical activation loop at the C-terminus (Figure S1). In addition, we analyzed the sequences of all MKK4/MKK5 copies from B. napus, B. rapa, B. oleracea, and A. thaliana. Based on phylogenetic analysis (Figure S2), we found that BnaA01.MKK5 was more closely related to BraA01.MKK5, while BnaC01.MKK5 was closer to BolC04.MKK5; the BnaA01.MKK5 copy was inherited from $B$. rapa, while the BnaC01.MKK5 copy was inherited from B. oleracea. Similarly, BnaA03.MKK5 and BnaC03.MKK5 were orthologs of those in the B. rapa and B. oleracea genome, respectively, and were also parahomologs in the $\mathrm{A}$ and $\mathrm{C}$ subgenomes of B. napus. In general, BnaA01.MKK5, BnaC01.MKK5, BnaA03.MKK5, and BnaC03.MKK5 were orthologs of AtMKK5. In Arabidopsis, MKK4 and MKK5 are two closely related MKKs that are functionally interchangeable with NtMEK2 in activating downstream MPKs [16]. The independent roles of AtMKK4 and AtMKK5 have not been revealed. Gene editing mediated by the CRISPR/Cas system has been successful and widely used, especially in targeting functional redundant genes. The abscisic acid (ABA) receptor family (PYL) consists of 14 genes with redundant functions in the model plant Arabidopsis [40]. Miao et al. generated pyl mutants with seven genes edited in rice using the CRISPR/Cas9 system and identified their effects on growth [41]. With the continuous development of gene editing technology, the detailed function of $B$. napus genes will be further revealed by mutant creation with multiple copies, including BnMKK4 and BnMKK5. In this work, we obtained bnmpk3 mutants that were edited on two copies and resulted in the deletion of the functional domain.

The Brassica species experienced a triploidization event in its evolution history, resulting in genome duplications of B. rapa and B. oleracea compared with Arabidopsis [1,42]. In addition, due to the fact that $B$. napus is a heterologous tetraploid derived from $B$. rapa and $B$. oleracea, in general, there are more than two homologs of one $A$. thaliana gene in the $B$. napus genome. However, MPK3 has only two copies in B. napus and one copy in $B$. rapa or B. oleracea (Figure $3 \mathrm{~A}$ ), meaning that this gene is alienated in the process of Brassica triploidization. Genome duplication is a driving force of plant evolution and is the primary way in which plants adapt to their natural environment [43]. In fact, not all duplicated genes inherited normal biological functions during genome evolution history; most duplicated gene pairs experienced pseudogenization or gene losses, whereas a few duplicate gene pairs were retained [44]. Gene loss is almost always accompanied by genome duplication. In addition, there are two homologs of AtMPK6 in the A or C subgenome, respectively, following the polyploid event of Brassica. These Brassica napus MPK6 are highly similar with BnaA06.MPK3 and BnaC03.MPK3, sharing conserved sub-domains and key motifs (Figure S3). AtMPK3/AtMPK6 is involved in biotic and abiotic stress, and AtMPK6 is also involved in plant growth and development. AtMPK3 and AtMPK6 are both 
phosphorylation substrates of AtMKK4/AtMKK5, showing functional redundancy [15-18]. This contributes to survival stability and prevent complete function loss in the case of gene mutation.

Phosphorylation-activated MAPKs act on downstream substrates, and transcription factors are widely reported to be phosphorylated by MAPK cascade. In Arabidopsis, activated MPK3/6 can directly phosphorylate downstream substrates, such as WRKY33, ERF104, and ICE1 [45-47]. The constitutively activated MKK4 ${ }^{\mathrm{DD}} / \mathrm{MKK} 5^{\mathrm{DD}}$ is used as an upstream activator of the MAPK cascade in the in vitro phosphorylation assay of MPK3/6 on its substrate $[46,47]$. In this study, BnaA03.MKK5 ${ }^{\mathrm{DD}}$ is a proved working activator and can be effectively used in the related research on MAPK cascade in B. napus.

In conclusion, we developed a detailed MAPK cascade module mediated by BnaA03.MKK5-BnaA06.MPK3/BnaC03.MPK3 in B. napus and proved that this module positively contributed to $S$. sclerotiorum resistance of B. napus. All B. napus MKK5 and MPK3 copies showed large-scale sequence identity, indicating that there is likely to be functional redundancy among copies or closely related members. Subsequently, it was identified that the BnaA03.MKK5, BnaA06.MPK3, and BnaC03.MPK3 copies were induced by Sclerotinia infection. Both BnaA06.MPK3 and BnaC03.MPK3 interacted with BnaA03.MKK5 and were directly phosphorylated by the upstream BnaA03.MKK5. This study not only reveals a detailed MAPK module, but also determines the function of the detailed MAPK cascade copies, laying the foundation for the further research of the response mechanisms against S. sclerotiorum in B. napus.

\section{Materials and Methods}

\subsection{Plant Materials, Growth Conditions and Plasmid Construction}

The B. napus varietie Jia 9709 were used to establish transgenic lines, including BnaA03.MKK5-OE, BnaA06.MPK3-OE, BnaC03.MPK3-OE, and BnaMPK3-CR. Wild-type and transgenic oilseed rape plants were grown in a field at the Wuhan experimental base for seed propagation. For oilseed rape leaf inoculation and Nicotiana benthamiana growth, seedlings were grown in a greenhouse $\left(22^{\circ} \mathrm{C}, 16 \mathrm{~h}\right.$ day $/ 8 \mathrm{~h}$ night $)$. The full-length coding sequences of BnaA03.MKK5 (BnaA03g36120D), BnaA06.MPK3 (BnaA06g18440D), and BnaC03.MPK3 (BnaC03g55440D) were driven by CaMV35S to generate overexpression constructs; the BnaMPK3 gene editing construct mediated by CRISPR/Cas9 was designed as previously described [48], and the sgRNA of targeted genes was designed using CRISPR-P (http:/ / cbi.hzau.edu.cn/cgi-bin/CRISPR, 23 February 2022). The recombinant plasmids were confirmed via sequencing and were introduced into Agrobacterium tumefaciens strain GV3101 and transferred to Jia 9709 in accordance with the previously mentioned method [49]. The primers for the constructs are listed in Table S2.

\subsection{RNA Extraction and $q R T-P C R$}

RNA was isolated from transgenic lines and WT plants (treated with pathogens) using the RNAprep pure plant kit (TIANGEN, DP441, Beijing, China). Then, $1 \mu \mathrm{g}$ of total RNA was used for cDNA synthesis using the RevertAid ${ }^{\mathrm{TM}}$ First Strand cDNA Synthesis Kit (Fermentas, \#K1622, Waltham, MA, USA). qRT-PCR analysis was performed with the CFX96 ${ }^{\mathrm{TM}}$ Real-Time system (Bio-Rad, Berkeley, CA, USA) using the SYBR Green Realtime PCR Master Mix (TOYOBO, QPK-201, Osaka, Japan). BnACTIN2 was used as a control to normalize expression levels according to the $2^{-\Delta \Delta C T}$ method. The primers used in the qRT-PCR assays are given in Table S2.

\subsection{Pathogen Inoculation and Lesion Measurement}

S. sclerotiorum A367 was used for inoculation and was cultured on potato dextrose agar medium (20\% potato, $2 \%$ dextrose and $1.5 \%$ agar) at $22{ }^{\circ} \mathrm{C}$ in the dark. The latest fully unfolded leaves were removed from oilseed rape plants of approximately 6 weeks, and the detached leaves were placed on soaked gauze. Then, the mycelial side of the fungus agar block (7 mm in diameter) was attached to the leaf surface. The inoculated leaves were 
covered with plastic films to maintain moisture at $22{ }^{\circ} \mathrm{C}$. After $48 \mathrm{~h}$ of inoculation, the images of inoculated leaves were taken and the lesion area was measured using ImageJ software.

\subsection{Sequence Alignment and Phylogenetic Analysis}

Sequence analysis was based on B. napus reference genome Darmor-bzh V5, the BnTIR (http:/ / yanglab.hzau.edu.cn/BnTIR, 23 February 2022) database and The Arabidopsis Information Resource (TAIR, TAIR 10) database. Sequence alignments were conducted using the ClustalW program in MEGA software (MEGA_11.0.10), and the results were displayed using the GENEDOC software (GeneDoc 2.7.0.0) with manual editing. The unrooted phylogenetic tree was constructed using the Maximum Likelihood method with 1000 replicates for bootstrap analysis, and MEGA software was used to display the result.

\subsection{Yeast Two-Hybrid (Y2H) Assay}

The $\mathrm{Y} 2 \mathrm{H}$ assays were performed following the manufacturer's protocol using the Matchmaker GAL4 Two-Hybrid System (Clontech, Shiga, Japan). The full-length BnaA03.MKK5 CDS was cloned into the pGADT7 vector, and the full-length CDSs of BnaA06.MPK3 and BnaC03.MPK3 were inserted into the pGBKT7 vector. To test proteinprotein interactions, the recombinant pGADT7 and pGBKT7 constructs were co-transformed into an AH109 yeast strain. Yeast cells were grown on selective dropout medium SD/-Trp Leu (lacking Trp and Leu) and SD/-Trp Leu His Ade (lacking Trp, Leu, His and Ade). The construct primers are listed in Table S2.

\subsection{In Vivo Co-Immunoprecipitation (CoIP) Assay}

The coding sequences of BnaA06.MPK3 and BnaC03.MPK3 were cloned into the pH7LIC6.0 vector to generate GFP fusion proteins, and the coding sequence of BnaA03.MKK5 was cloned into the $p H 7 L I C 4.1$ vector to generate GFP fusion proteins. The recombinant GFP and FLAG constructs in A. tumefaciens strain GV3101 were transiently co-expressed in 4 -week-old $N$. benthamiana leaves for 3 days. An amount of $\sim 5 \mathrm{~g}$ of infiltrated leaves was sampled, and the total protein was extracted using $5 \mathrm{~mL}$ of lysis buffer $(50 \mathrm{mM}$ Tris- $\mathrm{HCl}$ pH 7.5, $150 \mathrm{mM} \mathrm{NaCl}, 1 \%$ Triton X-100, $5 \mathrm{mM}$ EDTA, 10\% glycerol, and $1 \times$ protease inhibitor (Roche, Cat. No. 11836153001, Mannheim, Germany). Then, the samples were incubated with $25 \mu \mathrm{L}$ of GFP-Trap-MA (Chromotek, gtma-20, Munich, Germany) at $4{ }^{\circ} \mathrm{C}$ for $1 \mathrm{~h}$, followed by being washed five times with washing buffer $(0.1 \%$ Triton $\mathrm{X}-100$ instead of $1 \%$ Triton $\mathrm{X}-100$ in lysis buffer). The coupled target proteins were eluted with SDS loading buffer and detected with anti-FLAG (Abclonal, AE005, Wuhan, China, 1:10,000) and anti-GFP (Abclonal, AE012, Wuhan, China, 1:10,000) antibodies. The construct primers are listed in Table S2.

\subsection{In Vitro Phosphorylation Assay}

The coding sequences of BnaA06.MPK3 and BnaC03.MPK3 were fused with GST tag ( $p$ GEX4T-1 plasmid was used), and the coding sequence of BnaA03.MKK5 ${ }^{\mathrm{DD}}$ was cloned into the $p E T 32 a$ plasmid $(6 \times$ His was fused in the N-terminus of MKK5). The recombinant GST-BnaA06.MPK3, GST-BnaC03.MPK3, and His-BnaA03.MKK5 ${ }^{\mathrm{DD}}$ proteins were expressed in Escherichia coli strain BL21. Cells were harvested after $16 \mathrm{~h}$ of incubation with $0.2 \mathrm{mM}$ isopropyl-beta-D-thiogalactopyranoside (IPTG) at $16{ }^{\circ} \mathrm{C}$, and then, they were lysed using a high-pressure cell disrupter (JNBIO, Guangzhou, China). The protein samples were purified using GST-Sefinose resin (BBI, C600031, Shanghai, China) and $\mathrm{Ni}^{2+}$ affinity resin (BBI, C600033, Shanghai, China). The BnaA03.MKK5BnaA06.MPK3/BnaC03.MPK3 phosphorylation assay was performed as previously described [50]. GST-tagged BnaA06.MPK3/BnaC03.MPK3 were incubated with His-tagged BnaA03.MKK5 ${ }^{\text {DD }}$ in the reaction buffer (50 $\mu \mathrm{M}$ ATP, $50 \mathrm{mM}$ HEPES pH 7.5, $10 \mathrm{mM} \mathrm{MgCl}_{2}$, and $2 \mathrm{mM}$ dithiothreitol) at $25^{\circ} \mathrm{C}$ for $1 \mathrm{~h}$. Phosphorylation signals were detected using anti-Phospho-p44/42 antibody (CST, 4370T, Danvers, MA, USA, 1:2000). The recombinant 
BnaA03.MKK5 ${ }^{\mathrm{DD}}$ was detected with anti-His antibody (Abclonal, AE003, Wuhan, China, 1:10,000). The recombinant BnaA06.MPK3 and BnaC03.MPK3 were detected with anti-GST antibody (Abclonal, AE001, Wuhan, China, 1:10,000). The construct primers are listed in Table S2.

Supplementary Materials: The following supporting information can be downloaded at: https: //www.mdpi.com/article/10.3390/plants11050609/s1. Figure S1: Sequence alignment of identified MPK4/5 copies in Brassica napus and Arabidopsis. Figure S2: Phylogenetic analysis showing the relationship between each copy of B. napus MKK5 and MKK4/5 in Brassica species, as well as MKK4/5 in Arabidopsis. Figure S3: Sequence alignment of B. napus MPK3/ 6 copies. Table S1: Lesion areas of infected oilseed rape leaves after $48 \mathrm{~h}$ incubation with S. Sclerotiorum. Table S2: Primers used in this study.

Author Contributions: J.T. directed the project. K.Z. and J.T. conceived and designed the research. K.Z., C.Z. and Z.W. performed the experiments and analyzed the data. All authors interpreted and discussed the results. K.Z. and Z.W. wrote the manuscript. K.Z., C.Z., Z.W., F.L. and J.T. edited and modified the manuscript. J.W., B.Y., J.S., C.M. and T.F. supervised the research. All authors have read and agreed to the published version of the manuscript.

Funding: This research was financed by the funding from the National Key Research and Development Program of China (2016YFD0100305), and the National Natural Science Foundation of China (31376120).

Institutional Review Board Statement: Not applicable.

Informed Consent Statement: Not applicable.

Data Availability Statement: The data that support the findings of this study are available in the Supplementary Materials of this article.

Acknowledgments: We thank Daohong Jiang (Huazhong Agriculture University) for providing S. sclerotiorum strains, Pugang Yu for sequence analysis, and Qiang Xin for manuscript modifying.

Conflicts of Interest: The authors declare no conflict of interest.

$\begin{array}{ll}\text { Abbreviations } & \\ \text { MAPK/MPK } & \text { Mitogen-activated protein kinase } \\ \text { MAPKK/MKK/MEK } & \text { Mitogen-activated protein kinase kinase (MAPK kinase) } \\ \text { MAPKKK/MEKK } & \text { Mitogen-activated protein kinase kinase kinase (MAPKK kinase) } \\ \text { MPK3 } & \text { Mitogen-activated protein kinase 3 } \\ \text { MKK5 } & \text { Mitogen-activated protein kinase kinase 5 } \\ \text { PAMP } & \text { Pathogen-associated molecular pattern } \\ \text { PTI } & \text { Pathogen-associated molecular pattern (PAMP)-triggered immunity } \\ \text { ETI } & \text { Effector-triggered immunity } \\ \text { CD site } & \text { Common docking site } \\ \text { WT } & \text { Wild-type } \\ \text { Bn/Bna } & \text { Brassica napus } \\ \text { At } & \text { Arabidopsis thaliana } \\ \text { DD } & \text { Conserved Ser/Thr were mutated to Asp } \\ \text { CaMV35S } & \text { Cauliflower mosaic virus 35S promoter } \\ \text { OE } & \text { Overexpression } \\ \text { CDS } & \text { Coding sequence } \\ \text { qRT-PCR } & \text { Quantitative real-time PCR } \\ \text { PAD3 } & \text { PHYTOALEXIN DEFICIENT 3 } \\ \text { CYP71A13 } & \text { CYTOCHROME P450, FAMILY 71, SUBFAMILY A, POLYPEPTIDE 13 } \\ \pm \text { SD } & \pm \text { standard deviation } \\ \text { Y2H } & \text { Yeast two-hybrid } \\ \text { AD } & \text { Activation domain } \\ \text { BD } & \text { Binding domain } \\ \text { SD/-Trp Leu } & \text { Selective dropout medium lacking Trp and Leu } \\ & \end{array}$




$\begin{array}{ll}\text { CoIP } & \text { co-immunoprecipitation } \\ \text { GST } & \text { Glutathione S-transferase } \\ \text { GFP } & \text { Green fluorescent protein } \\ \text { QTL } & \text { Quantitative genetic loci } \\ \text { C-terminus } & \text { Carboxyl-terminus } \\ \text { CRISPR } & \text { Clustered regularly interspaced short palindromic repeats } \\ \text { cDNA } & \text { Complementary DNA }\end{array}$

\section{References}

1. Chalhoub, B.; Denoeud, F.; Liu, S.; Parkin, I.A.; Tang, H.; Wang, X.; Chiquet, J.; Belcram, H.; Tong, C.; Samans, B. Early allopolyploid evolution in the post-Neolithic Brassica napus oilseed genome. Science 2014, 345, 950-953. [CrossRef] [PubMed]

2. Kharbanda, P.; Tewari, J. Integrated management of canola diseases using cultural methods. Can. J. Plant Pathol. 1996, 18, 168-175. [CrossRef]

3. Derbyshire, M.; Denton-Giles, M. The control of sclerotinia stem rot on oilseed rape (Brassica napus): Current practices and future opportunities. Plant Pathol. 2016, 65, 859-877. [CrossRef]

4. Bolton, M.D.; Thomma, B.P.; Nelson, B.D. Sclerotinia sclerotiorum (Lib.) de Bary: Biology and molecular traits of a cosmopolitan pathogen. Mol. Plant Pathol. 2006, 7, 1-16. [CrossRef]

5. Dangl, J.L.; Jones, J.D. Plant pathogens and integrated defence responses to infection. Nature 2001, 411, 826-833. [CrossRef]

6. Jones, J.D.; Dangl, J.L. The plant immune system. Nature 2006, 444, 323-329. [CrossRef]

7. Bigeard, J.; Colcombet, J.; Hirt, H. Signaling mechanisms in pattern-triggered immunity (PTI). Mol. Plant 2015, 8, 521-539. [CrossRef]

8. Cui, H.; Tsuda, K.; Parker, J.E. Effector-triggered immunity: From pathogen perception to robust defense. Annu. Rev. Plant Biol. 2015, 66, 487-511. [CrossRef]

9. Dóczi, R.; Okrész, L.; Romero, A.; Paccanaro, A.; Bögre, L. Exploring the evolutionary path of plant MAPK networks. Trends Plant Sci. 2012, 17, 518-525. [CrossRef]

10. Ichimura, K.; Shinozaki, K.; Tena, G.; Sheen, J.; Henry, Y.; Champion, A.; Kreis, M.; Zhang, S.; Hirt, H.; Wilson, C. Mitogenactivated protein kinase cascades in plants: A new nomenclature. Trends Plant Sci. 2002, 7, 301-308.

11. Meng, X.; Zhang, S. MAPK cascades in plant disease resistance signaling. Annu. Rev. Phytopathol. 2013, 51, 245-266. [CrossRef] [PubMed]

12. Kong, Q.; Qu, N.; Gao, M.; Zhang, Z.; Ding, X.; Yang, F.; Li, Y.; Dong, O.; Chen, S.; Li, X.; et al. The MEKK1-MKK1/MKK2-MPK4 kinase cascade negatively regulates immunity mediated by a mitogen-activated protein kinase kinase kinase in Arabidopsis. Plant Cell 2012, 24, 2225-2236. [CrossRef] [PubMed]

13. Teige, M.; Scheikl, E.; Eulgem, T.; Dóczi, R.; Ichimura, K.; Shinozaki, K.; Dangl, J.; Hirt, H. The MKK2 pathway mediates cold and salt stress signaling in Arabidopsis. Mol. cell 2004, 15, 141-152. [CrossRef] [PubMed]

14. Takahashi, F.; Yoshida, R.; Ichimura, K.; Mizoguchi, T.; Seo, S.; Yonezawa, M.; Maruyama, K.; Yamaguchi-Shinozaki, K.; Shinozaki, $\mathrm{K}$. The mitogen-activated protein kinase cascade MKK3-MPK6 is an important part of the jasmonate signal transduction pathway in Arabidopsis. Plant Cell 2007, 19, 805-818. [CrossRef] [PubMed]

15. Asai, T.; Tena, G.; Plotnikova, J.; Willmann, M.; Chiu, W.; Gomez-Gomez, L.; Boller, T.; Ausubel, F.; Sheen, J. MAP kinase signalling cascade in Arabidopsis innate immunity. Nature 2002, 415, 977-983. [CrossRef] [PubMed]

16. Ren, D.; Yang, H.; Zhang, S. Cell death mediated by MAPK is associated with hydrogen peroxide production in Arabidopsis. J. Biol. Chem. 2002, 277, 559-565. [CrossRef] [PubMed]

17. Wang, H.; Ngwenyama, N.; Liu, Y.; Walker, J.; Zhang, S. Stomatal development and patterning are regulated by environmentally responsive mitogen-activated protein kinases in Arabidopsis. Plant Cell 2007, 19, 63-73. [CrossRef]

18. Cho, S.; Larue, C.; Chevalier, D.; Wang, H.; Jinn, T.; Zhang, S.; Walker, J. Regulation of floral organ abscission in Arabidopsis thaliana. Proc. Natl. Acad. Sci. USA 2008, 105, 15629-15634. [CrossRef]

19. Zhou, C.; Cai, Z.; Guo, Y.; Gan, S. An Arabidopsis mitogen-activated protein kinase cascade, MKK9-MPK6, plays a role in leaf senescence. Plant Physiol. 2009, 150, 167-177. [CrossRef]

20. Kim, K.; Min, J.; Dickman, M. Oxalic acid is an elicitor of plant programmed cell death during Sclerotinia sclerotiorum disease development. Mol. Plant-Microbe Interact. MPMI 2008, 21, 605-612. [CrossRef]

21. Liu, F.; Wang, M.; Wen, J.; Yi, B.; Shen, J.; Ma, C.; Tu, J.; Fu, T. Overexpression of barley oxalate oxidase gene induces partial leaf resistance to Sclerotinia sclerotiorum in transgenic oilseed rape. Plant Pathol. 2015, 64, 1407-1416. [CrossRef]

22. Amselem, J.; Cuomo, C.A.; van Kan, J.A.; Viaud, M.; Benito, E.P.; Couloux, A.; Coutinho, P.M.; de Vries, R.P.; Dyer, P.S.; Fillinger, S.; et al. Genomic analysis of the necrotrophic fungal pathogens Sclerotinia sclerotiorum and Botrytis cinerea. PLoS Genet. 2011, 7 e1002230. [CrossRef] [PubMed]

23. Wang, Z.; Wan, L.; Xin, Q.; Chen, Y.; Zhang, X.; Dong, F.; Hong, D.; Yang, G. Overexpression of OsPGIP2 confers Sclerotinia sclerotiorum resistance in Brassica napus through increased activation of defense mechanisms. J. Exp. Bot. 2018, 69, 3141-3155. [CrossRef] [PubMed]

24. Wang, Z.; Fang, H.; Chen, Y.; Chen, K.; Li, G.; Gu, S.; Tan, X. Overexpression of BnWRKY33 in oilseed rape enhances resistance to Sclerotinia sclerotiorum. Mol. Plant Pathol. 2014, 15, 677-689. [CrossRef] 
25. Liu, F.; Li, X.; Wang, M.; Wen, J.; Yi, B.; Shen, J.; Ma, C.; Fu, T.; Tu, J. Interactions of WRKY15 and WRKY33 transcription factors and their roles in the resistance of oilseed rape to Sclerotinia infection. Plant Biotechnol. J. 2018, 16, 911-925. [CrossRef]

26. Wang, Z.; Bao, L.; Zhao, F.; Tang, M.; Chen, T.; Li, Y.; Wang, B.; Fu, B.; Fang, H.; Li, G.; et al. BnaMPK3 Is a Key Regulator of Defense Responses to the Devastating Plant Pathogen in Oilseed Rape. Front. Plant Sci. 2019, 10, 91. [CrossRef]

27. Wang, Z.; Mao, H.; Dong, C.; Ji, R.; Cai, L.; Fu, H.; Liu, S. Overexpression of Brassica napus MPK4 enhances resistance to Sclerotinia sclerotiorum in oilseed rape. Mol. Plant-Microbe Interact. MPMI 2009, 22, 235-244. [CrossRef]

28. Wang, Z.; Zhao, F.; Tang, M.; Chen, T.; Bao, L.; Cao, J.; Li, Y.; Yang, Y.; Zhu, K.; Liu, S.; et al. BnaMPK6 is a determinant of quantitative disease resistance against Sclerotinia sclerotiorum in oilseed rape. Plant Sci. Int. J. Exp. Plant Biol. 2020, $291,110362$. [CrossRef]

29. Stotz, H.U.; Sawada, Y.; Shimada, Y.; Hirai, M.Y.; Sasaki, E.; Krischke, M.; Brown, P.D.; Saito, K.; Kamiya, Y. Role of camalexin, indole glucosinolates, and side chain modification of glucosinolate-derived isothiocyanates in defense of Arabidopsis against Sclerotinia sclerotiorum. Plant J. 2011, 67, 81-93. [CrossRef]

30. Ahuja, I.; Kissen, R.; Bones, A.M. Phytoalexins in defense against pathogens. Trends Plant Sci. 2012, 17, 73-90. [CrossRef]

31. Mucha, S.; Heinzlmeir, S.; Kriechbaumer, V.; Strickland, B.; Kirchhelle, C.; Choudhary, M.; Kowalski, N.; Eichmann, R.; Hückelhoven, R.; Grill, E.; et al. The Formation of a Camalexin Biosynthetic Metabolon. Plant Cell 2019, 31, 2697-2710. [CrossRef] [PubMed]

32. Al-Shehbaz, I.A. A generic and tribal synopsis of the Brassicaceae (Cruciferae). Taxon 2012, 61, 931-954. [CrossRef]

33. Zhang, T.; Wang, N.; Wang, J.; Wang, Y.; Zhang, Y.; Sun, W.; Chang, Y.; Xia, H. Cloning and expression analysis of a BnMKK4 gene from Brassica napus. Plant Physiol. Commun. 2012, 48, 491-498.

34. Yang, K.; Liu, Y.; Zhang, S. Activation of a mitogen-activated protein kinase pathway is involved in disease resistance in tobacco. Proc. Natl. Acad. Sci. USA 2001, 98, 741-746. [CrossRef] [PubMed]

35. Su, J.; Zhang, M.; Zhang, L.; Sun, T.; Liu, Y.; Lukowitz, W.; Xu, J.; Zhang, S. Regulation of stomatal immunity by interdependent functions of a pathogen-responsive MPK3/MPK6 cascade and abscisic acid. Plant Cell 2017, 29, 526-542. [CrossRef] [PubMed]

36. Zhang, M.; Su, J.; Zhang, Y.; Xu, J.; Zhang, S. Conveying endogenous and exogenous signals: MAPK cascades in plant growth and defense. Curr. Opin. Plant Biol. 2018, 45, 1-10. [CrossRef]

37. Zhao, J.; Udall, J.A.; Quijada, P.A.; Grau, C.R.; Meng, J.; Osborn, T.C. Quantitative trait loci for resistance to Sclerotinia sclerotiorum and its association with a homeologous non-reciprocal transposition in Brassica napus L. Theor. Appl. Genet. 2006, 112, 509-516. [CrossRef]

38. Wei, L.; Jian, H.; Lu, K.; Filardo, F.; Yin, N.; Liu, L.; Qu, C.; Li, W.; Du, H.; Li, J. Genome-wide association analysis and differential expression analysis of resistance to Sclerotinia stem rot in Brassica napus. Plant Biotechnol. J. 2016, 14, 1368-1380. [CrossRef]

39. Zhang, F.; Huang, J.; Tang, M.; Cheng, X.; Liu, Y.; Tong, C.; Yu, J.; Sadia, T.; Dong, C.; Liu, L. Syntenic quantitative trait loci and genomic divergence for Sclerotinia resistance and flowering time in Brassica napus. J. Integr. Plant Biol. 2019, 61, 75-88. [CrossRef]

40. Park, S.Y.; Fung, P.; Nishimura, N.; Jensen, D.R.; Fujii, H.; Zhao, Y.; Lumba, S.; Santiago, J.; Rodrigues, A.; Chow, T.F.; et al. Abscisic acid inhibits type 2C protein phosphatases via the PYR/PYL family of START proteins. Science 2009, 324, $1068-1071$. [CrossRef]

41. Miao, C.; Xiao, L.; Hua, K.; Zou, C.; Zhao, Y.; Bressan, R.A.; Zhu, J.K. Mutations in a subfamily of abscisic acid receptor genes promote rice growth and productivity. Proc. Natl. Acad. Sci. USA 2018, 115, 6058-6063. [CrossRef] [PubMed]

42. Wang, X.; Wang, H.; Wang, J.; Sun, R.; Wu, J.; Liu, S.; Bai, Y.; Mun, J.H.; Bancroft, I.; Cheng, F.; et al. The genome of the mesopolyploid crop species Brassica rapa. Nat. Genet. 2011, 43, 1035-1039. [CrossRef] [PubMed]

43. Soltis, P.S.; Liu, X.; Marchant, D.B.; Visger, C.J.; Soltis, D.E. Polyploidy and novelty: Gottlieb's legacy. Philos. Trans. R. Soc. B Biol. Sci. 2014, 369, 1648. [CrossRef] [PubMed]

44. Liu, H.J.; Tang, Z.X.; Han, X.M.; Yang, Z.L.; Zhang, F.M.; Yang, H.L.; Liu, Y.J.; Zeng, Q.Y. Divergence in Enzymatic Activities in the Soybean GST Supergene Family Provides New Insight into the Evolutionary Dynamics of Whole-Genome Duplicates. Mol. Biol. Evol. 2015, 32, 2844-2859. [CrossRef] [PubMed]

45. Bethke, G.; Unthan, T.; Uhrig, J.F.; Pöschl, Y.; Gust, A.A.; Scheel, D.; Lee, J. Flg22 regulates the release of an ethylene response factor substrate from MAP kinase 6 in Arabidopsis thaliana via ethylene signaling. Proc. Natl. Acad. Sci. USA 2009, 106, 8067-8072. [CrossRef] [PubMed]

46. Mao, G.; Meng, X.; Liu, Y.; Zheng, Z.; Chen, Z.; Zhang, S. Phosphorylation of a WRKY transcription factor by two pathogenresponsive MAPKs drives phytoalexin biosynthesis in Arabidopsis. Plant Cell 2011, 23, 1639-1653. [CrossRef]

47. Li, H.; Ding, Y.; Shi, Y.; Zhang, X.; Zhang, S.; Gong, Z.; Yang, S. MPK3- and MPK6-Mediated ICE1 Phosphorylation Negatively Regulates ICE1 Stability and Freezing Tolerance in Arabidopsis. Dev. Cell 2017, 43, 630-642.e634. [CrossRef]

48. Xing, H.; Dong, L.; Wang, Z.; Zhang, H.; Han, C.; Liu, B.; Wang, X.; Chen, Q. A CRISPR/Cas9 toolkit for multiplex genome editing in plants. BMC Plant Biol. 2014, 14, 327. [CrossRef]

49. Zhang, K.; He, J.; Liu, L.; Xie, R.; Qiu, L.; Li, X.; Yuan, W.; Chen, K.; Yin, Y.; Kyaw, M.M.M. A convenient, rapid and efficient method for establishing transgenic lines of Brassica napus. Plant Methods 2020, 16, 43. [CrossRef]

50. Guo, T.; Lu, Z.Q.; Shan, J.X.; Ye, W.W.; Dong, N.Q.; Lin, H.X. ERECTA1 Acts Upstream of the OsMKKK10-OsMKK4-OsMPK6 Cascade to Control Spikelet Number by Regulating Cytokinin Metabolism in Rice. Plant Cell 2020, 32, 2763-2779. [CrossRef] 